\title{
Optimal Solution of Multi-Choice Mathematical Programming Problem Using a New Technique
}

\author{
Tarek A. Khalil1, Yashpal Singh Raghav ${ }^{2}$, N. Badra ${ }^{3}$ \\ ${ }^{1}$ Mathematics Department, Higher Institute of Engineering and Technology, New Cairo Academy, Cairo, Egypt \\ ${ }^{2}$ Department of Mathematics, Faculty of Science, Jazan University, Jazan, KSA \\ ${ }^{3}$ Department of Physics and Engineering Mathematics, Faculty of Engineering, Ain Shams University, Cairo, Egypt \\ Email:tibrahimg@gmail.com, ”yraghav@jazanu.edu.sa,n_b_eng_ainshams@yahoo.com
}

Received 28 January 2016; accepted 7 March 2016; published 10 March 2016

Copyright (C) 2016 by authors and Scientific Research Publishing Inc.

This work is licensed under the Creative Commons Attribution International License (CC BY). http://creativecommons.org/licenses/by/4.0/

(c) (i) Open Access

\section{Abstract}

The study deals with the multi-choice mathematical programming problem, where the right hand side of the constraints is multi-choice in nature. However, the problem of multi-choice linear programming cannot be solved directly by standard linear or nonlinear programming techniques. The aim of this paper is to transform such problems to a standard mathematical linear programming problem. For each constraint, exactly one parameter value is selected out of a multiple number of parameter values. This process of selection can be established in different ways. In this paper, we present a new simple technique enabling us to handle such problem as a mixed integer linear programming problem and consequently solve them by using standard linear programming software. Our main aim depends on inserting a specific number of binary variables and using them to construct a linear combination which gives just one parameter among the multiple choice values for each choice of the values of the binary variables. A numerical example is presented to illustrate our analysis.

\section{Keywords}

Multi-Choice Mathematical Programming, Transformation Technique, Optimization

\section{Introduction}

Most of real life and industrial problems involve a process called optimization, which means finding the ${ }^{*}$ Corresponding author.

How to cite this paper: Khalil, T.A., Raghav, Y.S. and Badra, N. (2016) Optimal Solution of Multi-Choice Mathematical Programming Problem Using a New Technique. American Journal of Operations Research, 6, 167-172. 
maximum or minimum value of some quantity called objective function, subject to a system of linear inequalities or equalities called constraints. The graph of the system of constraints is called the feasible region and the optimal values of the objective function occur at vertices of the feasible region. Many research papers handle this problem from more than one point of view and gave very helpful and considerable results.

Linear programming is frequently applied in real-life problems and therefore it is very important to introduce new tools in the approach that allow the model to fit into the real-life problems. Linear programming was developed by Dantzig [5]. Linear programming has been applied to various decision making problems. Most extensively it is used in business and some industrial engineering problems. Some industries that use linear programming models include transportation, production planning and scheduling, energy, telecommunications, and manufacturing. It has been proved useful in modeling diverse types of problems in planning, routing, scheduling, assignment, and design. Any linear programming model representing real-world situations involves a lot of parameters whose values are assigned by the experts, and in the conventional approach, they are required to fix an exact value to the aforementioned parameters. However, both experts and the decision-maker frequently do not precisely know the value of those parameters, in most cases they depend only on statistical inference from past data and their stability is doubtful, so the parameters of the problem are usually defined by the decision maker in a uncertain way or by means of language statement parameters. Approaches to decision making under uncertainty have followed a variety of modeling philosophies, including minimization of expected value of loss, minimization of deviations from goals, minimization of costs and maximization of profits. The main approaches to decision making under uncertainty include stochastic and fuzzy programming. However, in some cases it is believed that the parameters or coefficients in the decision making problems are multi-choice in nature.

Recently, a considerable work and many attractive results have appeared in this research area, see [1]-[17] and references therein. Hiller and Lieberman [4] and Ravindran et al. [1] have considered a mathematical model in which an appropriate constraint is to be chosen using binary variables. The number of binary variables required for a constraint is same as the total number of choices for that constraint. Biswal and Acharya [8] gave new approach for treating the multi-choice linear programming problem. they used multiplicative terms of binary variables. Also, in [9], interpolating polynomial associated with the parameter values is used to replace the multi-choices values in the right hand side of the constrains. In the end of the paper we discuss these two techniques in more details.

Chang [2] has recently proposed a new technique for modeling the multi-choice goal programming. In other paper [3], he replaces multiplicative terms of binary variable by a continuous variable. An alternative approach for solving such kind of problem is chance constrained programming by Birge and Louveaux [6] and Sahoo \& Biswal [10]. Many of the developments in the area of fuzzy mathematical programming are based on the paper written by Bellman and Zadeh [11].

This paper is organized as follows: The first section is devoted for introduction and historical survey of the subject, in the second section, we give the mathematical model of the general multi-choice linear programming problem. While in Section 3 we present our technique and explain how it works to transform the multi-choice linear programming problem to a standard linear programming one, in addition, we give our main results in a compact mathematical form. In Section 4, we offer a numerical example to show the procedure of our technique. We conclude the paper by a brief discussion to compare our result with other results given by Biswal and Acharya [8] [9]. We end the paper by some helpful references.

\section{Mathematical Model}

The mathematical model of a multi-choice linear programming problem is presented as:

Find $X=\left(x_{1} ; x_{2} ; \cdots ; x_{n}\right)$ so as to:

$$
\max : Z=\sum_{j=1}^{n} c_{j} x_{j}
$$

subject to:

$$
\begin{gathered}
\sum_{j=1}^{n} a_{i j} x_{j} \leq\left\{b_{i 1}, b_{i 2}, \cdots, b_{i k_{i}}\right\}, i=1,2, \cdots, m \\
x_{j} \geq 0, \quad j=1,2, \cdots, n
\end{gathered}
$$


The right hand side of each constraint (2) has a set of $k_{i}$ number of goals where only one goal is to be selected. To solve the multi-choice linear programming problem (1)-(3) it is necessary to transform the problem to a standard mathematical programming problem. In the coming section a general linear transformation is presented for all cases. our technique works not only for small values of $k_{i}$ but also for the large values.

\section{Linear Transformation}

Let $b_{i 1}, b_{i 2}, \cdots, b_{i k_{i}}$ denote the set of values (choices) in the right hand side of the $i^{\text {th }}$ constrain with total number $k_{i}$.

Setting:

$$
K:=\text { Least Common Multiple of the numbers }\left\{k_{1}, k_{2}, \cdots, k_{m}\right\}
$$

In order to achieve our main aim of selecting just one value among the $k_{i}$ choices, our idea depend on inserting a set of $K$ binary variables, namely: $z_{1}, z_{2}, \cdots, z_{K}$ to construct a set of $r_{i}:=\frac{K}{k_{i}}$ linear combinations in the following form:

$$
\begin{aligned}
& L_{i 1}:=b_{i 1} z_{1}+b_{i 2} z_{2}+\cdots+b_{i k_{i}} z_{k_{i}}=\sum_{\ell=1}^{k_{i}} b_{i \ell} z_{\ell} \\
& L_{i 2}:=b_{i 1} z_{k_{i}+1}+b_{i 2} z_{k_{i}+2}+\cdots+b_{i k_{i}} z_{2 k_{i}}=\sum_{\ell=1}^{k_{i}} b_{i \ell} z_{k_{i}+\ell} \\
& L_{i 3}:=b_{i 1} z_{2 k_{i}+1}+b_{i 2} z_{2 k_{i}+2}+\cdots+b_{i k_{i}} z_{3 k_{i}}=\sum_{\ell=1}^{k_{i}} b_{i \ell} z_{2 k_{i}+\ell} \\
& \vdots \\
& L_{i, r_{i}}:=b_{i 1} z_{\left(r_{i}-1\right) k_{i}+1}+b_{i 2} z_{\left(r_{i}-1\right) k_{i}+2}+\cdots+b_{i k_{i}} z_{r_{i} k_{i}}=\sum_{\ell=1}^{k_{i}} b_{i, \ell} z_{\left(r_{i}-1\right) k_{i}+\ell}
\end{aligned}
$$

Finally, we replace the right hand side of the $i^{\text {th }}$ constrain by the expression:

$$
\mathbb{L}_{i}:=\sum_{j=1}^{r_{i}} L_{i j}=\sum_{j=1}^{r_{i}}\left\{\sum_{\ell=1}^{k_{i}} b_{i, \ell} Z_{(j-1) k_{i}+\ell}\right\}
$$

Now, we can rewrite the mathematical model (1)-(3) in more convenient form as:

Find $X=\left(x_{1} ; x_{2} ; x_{3} ; \cdots ; x_{n}\right)$ so as to:

$$
\max : Z=\sum_{j=1}^{n} c_{j} x_{j}
$$

subject to:

$$
\begin{gathered}
\sum_{j=1}^{n} a_{i j} x_{j} \leq \mathbb{L}_{i}, \quad i=1,2, \cdots, m \\
Z_{p}=0 \backslash 1, \quad p=1,2, \cdots, K \\
\sum_{p=1}^{K} z_{p}=1, \quad x_{j} \geq 0, \quad j=1,2, \cdots, n
\end{gathered}
$$

where $\mathbb{L}_{i}$ given in (6). The restriction $\left(\sum_{p=1}^{K} z_{p}=1\right)$ mentioned in (10) implies that one and only one of the $K$ 's binary variable will take the value one while the remaining $(K-1)^{\prime} s$ will take the value zero which ensures the achievement of our main aim of selecting a unique parameter value among the $k_{i}^{\prime} s$ values in the right hand side of each constrain.

\section{Illustrative Numerical Example}

A factory produce three different types of washing machines A, B, and C. Producing of one washing machine of 
type A requires 10 hours general labor, 5 technical hours, and 1 packing hour. While the washing machine of type B requires 4 hours general labor, 7 technical hours, and 2 packing hours. and the washing machine of type $\mathrm{C}$ requires 5 hours general labor, 2 technical hours, and 3 packing hours. The factory can afford up to 900 or 1000 or 1100 hours of general labor, up to 1000 or 1450 or 1600 or 2000 hours of technical labor, and up to 300 or 400 packing hours per week. A washing machine of type A, type B, and type C earns a profit of $£ 130, £ 100$, and $£ 80$ respectively. The factory decided to produce at least 20 washing machines of type $C$ per week. How many washing machines per week should the factory produce from each type to maximize profit?

Let $x_{1}, x_{2}$, and $x_{3}$ denote the number of produced washing machines of type A, B, and C per week respectively.

The following table summarize the information above:

\begin{tabular}{|c|c|c|c|c|}
\hline Type & A & B & $\mathrm{C}$ & Total available Hs. \\
\hline General Labor & 10 Hs. & 4 Hs. & 5 Hs. & 900 or 1000 or 1100 \\
\hline Technical Time & 5 Hs. & 7 Hs. & 2 Hs. & 1000 or 1450 or 1600 or 2000 \\
\hline Packing Time & $1 \mathrm{H}$. & 2 Hs. & 3 Hs. & 300 or 400 \\
\hline Profit & 130 & 100 & 80 & \\
\hline \# Wash. Mach. & $x_{1}$ & $x_{2}$ & $x_{3}$ & \\
\hline
\end{tabular}

Since the manufacturer wants to maximize the profit, so, we have the following mathematical multi-choice problem:

$$
\operatorname{Max}: f=130 x_{1}+100 x_{2}+80 x_{3}
$$

subject to the constrains:

$$
\begin{gathered}
10 x_{1}+4 x_{2}+5 x_{3} \leq\{900,1000,1100\} \\
5 x_{1}+7 x_{2}+2 x_{3} \leq\{1000,1450,1600,2000\} \\
x_{1}+2 x_{2}+3 x_{3} \leq\{300,400\} \\
x_{1}, x_{2} \geq 0, x_{3} \geq 20
\end{gathered}
$$

Applying the above technique in (5) and (6), we can transform the given multi-choice programming problem to a mixed integer linear programming problem. Referring to the data above, we have $k_{1}=3, k_{2}=4, k_{3}=2$. So, $\mathbb{K}=12$ which denotes the least common multiple of the integers $(3,4,2)$. Therefore, we insert 12 binary variables in the right hand side of the three constraints and rewrite the problem in the the new form:

$$
\text { Max : } f=130 x_{1}+100 x_{2}+80 x_{3}
$$

subject to:

$$
\begin{aligned}
& 10 x_{1}+4 x_{2}+5 x_{3} \leq 900 z_{1}+1000 z_{2}+1100 z_{3}+900 z_{4}+1000 z_{5}+1100 z_{6} \\
&+ 900 z_{7}+1000 z_{8}+1100 z_{9}+900 z_{10}+1000 z_{11}+1100 z_{12} \\
& 5 x_{1}+7 x_{2}+2 x_{3} \leq 1000 z_{1}+1450 z_{2}+1600 z_{3}+2000 z_{4}+1000 z_{5}+1450 z_{6} \\
&+ 1600 z_{7}+2000 z_{8}+1000 z_{9}+1450 z_{10}+1600 z_{11}+2000 z_{12} \\
& x_{1}+2 x_{2}+3 x_{3} \leq 300 z_{1}+400 z_{2}+300 z_{3}+400 z_{4}+300 z_{5}+400 z_{6} \\
&+ 300 z_{7}+400 z_{8}+300 z_{9}+400 z_{10}+300 z_{11}+400 z_{12} \\
& z_{p}=0 \backslash 1, \quad p=1,2, \cdots, 12 \\
& \sum_{p=1}^{12} z_{p}=1, x_{1}, x_{2} \geq 0, x_{3} \geq 20
\end{aligned}
$$

The above mathematical model is treated by either LINGO or MATLAB software. 
The corresponding Matlab code is:

$$
\begin{aligned}
& >f=[-130 ;-100 ;-80 ; 0 ; 0 ; 0 ; 0 ; 0 ; 0 ; 0 ; 0 ; 0 ; 0 ; 0 ; 0] \text {; } \\
& >A=[1045-900-1000-1100-900-1000-1100-900-1000-1100-900-1000-1100 \text {; } \\
& 572-1000-1450-1600-2000-1000-1450-1600-2000-1000-1450-1600-2000 \text {; } \\
& 123-300-400-300-400-300-400-300-400-300-400-300-400] \text {; } \\
& >b=\left[\begin{array}{lll}
0 & 0 & 0
\end{array}\right] \\
& >\text { Aeq }=\left[\begin{array}{lllll}
0 & 0 & 0 & 111111111111
\end{array}\right] \\
& >\text { beq }=1 \\
& >>\text { intcon }=(1: 15) \text {; } \\
& >l b=[0 ; 0 ; 20 ; 0 ; 0 ; 0 ; 0 ; 0 ; 0 ; 0 ; 0 ; 0 ; 0 ; 0 ; 0] \text {; } \\
& >>u b=\text { [inf ;inf ;inf } ; 1 ; 1 ; 1 ; 1 ; 1 ; 1 ; 1 ; 1 ; 1 ; 1 ; 1 ; 1] \text {; } \\
& >>[x, f v a l]=\text { intlinprog }(f \text {, intcon, } A, b, \text { Aeq, beq, } l b, u b)
\end{aligned}
$$

The solution is obtained as: $x_{1}=40, x_{2}=150$, and $x_{3}=20$ where the maximum profit is $£ 21,800$. The all binary variables are zeros except $z_{6}$ take the value one which means that the choice of the goals of first, second and third constraints are 1100 gal, 1450 gal and 400 hours respectively. One can follow the techniques in [8] [9] to obtain the same solution.

\section{Discussion and Conclusions}

Biswal and Acharya [8] treat the problem of multi-choice linear programming problem by replacing the right hand side of each constraint with a specific mathematical expressions involving the multi-choice values multiplied by polynomials of binary variables, those expressions change from one constraint to another according to the number of multi-choice values in that constraint. Moreover, some restrictions are imposed on the binary variable to obtain just one value in the right hand side of each constraint. In their other paper [9], they replaced the right hand side of each constraint with an interpolating polynomial more precisely, assuming that the $i^{\text {th }}$ constraint contains $k_{i}$ multi-choice values then the corresponding interpolating polynomial will be of degree $\leq\left(k_{i}-1\right)$ which gives just one value among the $k_{i}$ values corresponding to each value of the independent variable $z_{i}=0,1, \cdots, k_{i}-1$. The technique in this paper transforms the right hand side of each constraint to a linear combination with the same number of terms which enable us to treat the problem as a usual linear programming problem. Indeed, using different softwares we found that our technique gives the same results as [8] [9].

The aim of the article was to describe a new efficient technique for solving mathematical multi-choice problems the process depend on obtaining a number of linear mathematical expressions equal to the number of the multi-choice constrains and contain a specified number of binary variables. We tried to set the models as general as possible and that way make them applicable to any given mathematical multi-choice linear programming task. In practice it is possible to describe the majority of such problems. We can state that these models can serve as a simple and easy method for solving this type of mathematical problems using available softwares as Matlab and Lingo. Transforming the multi-choice problem to a linear problem is considered the main advantage of this method. The present method can be extended to a multi-objective multi-choice linear programming problem.

\section{Acknowledgements}

The authors are thankful to the editor in chief and the learned referees for their valuable suggestions regarding the improvement of this paper.

\section{References}

[1] Ravindran, A., Phillips, D.T. and Solberg, J.J. (1987) Operations Research Principles \& Practice. 2nd Edition, John Wiley, New York.

[2] Chang, C.-T. (2007) Multi-Choice Goal Programming. Omega, 35, 389-396. http://dx.doi.org/10.1016/j.omega.2005.07.009

[3] Chang, C.-T. (2008) Revised Multi-Choice Goal Programming. Applied Mathematical Modelling, 32, 2587-2595. http://dx.doi.org/10.1016/j.apm.2007.09.008 
[4] Hiller, F. and Lieberman, G. (1990) Introduction to Operations Research. McGraw-Hill, New York.

[5] Dantzig, G.B. (1963) Linear Programming and Extensions. Princeton University Press, Princeton.

[6] Birge, J.R. and Louveaux, F.V. (1997) Introduction to Stochastic Programming. Springer, New York.

[7] Tamiz, M., Jones, D. and Romero, C. (1998) Goal Programming for Decision Making: An Overview of the Current State-of-the-Art. European Journal of Operational Research, 111, 567-581. http://dx.doi.org/10.1016/S0377-2217(97)00317-2

[8] Biswal, M.P. and Acharya, S. (2009) Transformation of a Multi-Choice Linear Programming Problem. Applied Mathematics and Computation, 210, 182-188. http://dx.doi.org/10.1016/j.amc.2008.12.080

[9] Biswal, M.P. and Acharya, S. (2011) Solving Multi-Choice Linear Programming Problems by Interpolating Polynomials. Mathematical and Computer Modelling, 54, 1405-1412. http://dx.doi.org/10.1016/j.mcm.2011.04.009

[10] Sahoo, N.P. and Biswal, M.P. (2009) Computation of a Multi-Objective Production Planning Model with Probabilistic Constraints. International Journal of Computer Mathematics, 86, 185-198. http://dx.doi.org/10.1080/00207160701734207

[11] Bellman, R.E. and Zadeh, L.A. (1970) Decision-Making in a Fuzzy Environment. Management Science, 17, $141-161$. http://dx.doi.org/10.1287/mnsc.17.4.B141

[12] Yang, W.Y., Cao, W.W., Chung, T.-S. and Morris, J. (2005) Applied Numerical Methods Using MATLAB ${ }^{\circledR}$. John Wiley \& Sons, Inc., Hoboken. http://dx.doi.org/10.1002/0471705195

[13] Lee, C.-S. (2012) Multi-Objective Game-Theory Models for Conflict Analysis in Reservoir Watershed Management. Chemosphere, 87, 608-613.

[14] Zhang, G.-Q., Sun, Q.-B. and Wang, L. (2013) Noise Induced Enhancement of Network Reciprocity in Social Dilemmas. Chaos, Solitons \& Fractals, 51, 31-35. http://dx.doi.org/10.1016/j.chaos.2013.03.003

[15] Lu, J., Zhang, G., Ruan, D. and Wu, F. (2007) Multi-Objective Group Decision Making. Imperial College Press, London. http://dx.doi.org/10.1142/p505

[16] Zamarripa, M., Aguirre, A., Mendez, C. and Espuna, A. (2012) Integration of Mathematical Programming and Game Theory for Supply Chain Planning Optimization in Multi-Objective Competitive Scenarios. Computer Aided Chemical Engineering, 30, 402-406. http://dx.doi.org/10.1016/B978-0-444-59519-5.50081-2

[17] Jin, Q. and Wang, Z. (2014) Spontaneous Symmetry Breaking in Interdependent Networked Game. Scientific Reports, 4, 4095. http://dx.doi.org/10.1038/srep04095 